\title{
Muon capture in hydrogen and deuterium
}

\author{
Claude Petitjean - Representing the MuCap \\ and the MuSun collaborations
}

Published online: 26 August 2009

(C) Springer Science + Business Media B.V. 2009

\begin{abstract}
We report on a new generation of muon lifetime experiments at PSI to measure the nuclear muon capture rate in hydrogen and deuterium with $\leq 1 \%$ accuracy. The goals are to determine in $\mu p$ capture the induced pseudoscalar coupling $\mathrm{g}_{P}$ predicted in $\mathrm{HBchPT}$, and in $\mu d$ capture the axial two-body current term $\mathrm{L}_{1 A}$ described by modern EFT's. For the $\mu p$ experiment a hydrogen TPC was developed as active muon stop detector, surrounded by cylindrical wire chambers and a plastic hodoscope as electron detector. Ultra-high purity of the hydrogen isotope ${ }^{1} H_{1}$ at levels below $10^{-8}$ was achieved with a specially developed gas circulation and purification system, and with a novel isotope separation column. About $2 \cdot 10^{10}$ events were collected which are now in final analysis. Data from the first production run result in $\mathrm{g}_{P}=7.3 \pm 1.1$ in good agreement with theory. The $\mu d$ experiment is in development. It requires measurements in ultra-pure, cold deuterium gas at $\sim 30 \mathrm{~K}$. For this we are constructing a new Cryo-TPC.
\end{abstract}

Keywords Muon capture $\cdot$ Muon lifetime $\cdot$ Time projection chamber $\cdot$ Hydrogen

\section{Introduction}

A new generation of muon lifetime precision experiments has been introduced at PSI to measure the singlet rate $\Lambda_{S}$ of nuclear muon capture on the proton (MuCap experiment [1]), and the doublet capture rate $\Lambda_{D}$ on the deuteron (MuSun experiment [2]), to better than $1 \%$ accuracy:

$$
\begin{gathered}
\mu+p \rightarrow \mu p \rightarrow n+v_{\mu}, \\
\mu+d \rightarrow \mu d \rightarrow n+n+v_{\mu} .
\end{gathered}
$$

C. Petitjean $(\bowtie)$

Paul Scherrer Institut, 5232 Villigen, Switzerland

e-mail: claude.petitjean@psi.ch 
The goal of these experiments is to precisely determine fundamental constants of the weak interaction, particularly the induced pseudoscalar coupling constant $\mathrm{g}_{P}$ from the rate $\Lambda_{S}$ of reaction (1) which is predicted by heavy baryon chiral perturbation theory [3], and the axial two-body current term $\mathrm{L}_{1 A}$ from the rate $\Lambda_{D}$ of reaction (2) which is described by modern effective field theories $[4,5]$. The $\mathrm{L}_{1 A}$ parameter is of high astrophysical interest, because it calibrates the solar pp fusion reaction and also the $v+d$ reactions which are measured by the Sudbury Neutrino Observatory.

Since direct absolute rate measurements of the neutrons in the output channels are severely limited in accuracy, we have experimentally chosen the lifetime method which determines the capture rates from the difference of the $\mu^{-}$lifetimes in $\mu p$ $(\mu d), \lambda^{-}$, with respect to the lifetime of the free $\mu^{+}, \lambda^{+}$:

$$
\Lambda_{\text {capture }}=\lambda^{-}-\lambda^{+} \text {. }
$$

This method was first adopted at Saclay [6] in measurements with liquid hydrogen targets. However, mesomolecular physics, the formation of mesic molecules $p \mu p$ $(d \mu d)$, and subsequent processes, i.e. ortho-para transitions in $p \mu p, \mu d$ spin flip and fusion of the $d \mu d$ molecules, make the interpretation of the Saclay results difficult, because the spin populations cannot be determined precisely enough. We have avoided this problem by doing the MuCap measurements in low density gas, $\phi=$ 0.011 ( $\phi=1$ is the density of liquid hydrogen), where only $\sim 4 \%$ of the muons form p $\mu$ molecules, and we have developed a hydrogen time projection chamber (TPC) which acts as active target and allows to track each incoming muon to its stopping point. For the MuSun experiment on $\mu d$ capture a new Cryo-TPC is in development to operate in cold deuterium gas at density $\phi=0.05-0.10$, see chapter 3 .

\section{The MuCap experiment}

Figure 1 shows the TPC which was used in the $\mu p$ capture experiment. It is constructed from UHV materials (mainly metals, glass frames, ceramics) which can be baked out in order to achieve ultra-high gas purity. In a volume $30 \times 15 \times 12 \mathrm{~cm}^{3}$ a vertical electrical field of $2 \mathrm{kV} / \mathrm{cm}$ is applied. The electrons from ionizing particles drift down towards a MWPC plane. The amplified signals are read out in both plane coordinates while the vertical position is determined by the drift time $(0-24 \mu s)$. The TPC is sensitive to muons stopping in the volume, but also to reactions with charged particle emissions, e.g. nuclear recoils from muon capture processes on higher-Z elements. Such events were continuously monitored to control the level of impurities. Tracking the electrons from $\mu \rightarrow e$ decays with the outer detector back to the $\mu$ stop vertex allows clean identification of muon decays inside the sensitive TPC volume, while background and accidental events are highly suppressed.

A cut-out view of the MuCap apparatus is shown in Fig. 2. The TPC is mounted in an aluminum vessel filled with 10 bar of ultra-pure protium gas (deuterium depleted hydrogen) at $300 \mathrm{~K}$. Each muon stopping in the TPC is identified by scintillator $\mu \mathrm{SC}$ and wire chamber $\mu \mathrm{PC}$ at the entrance and by the track in the TPC. The decay electrons are detected by two cylindrical wire chambers ePC1 and ePC2 and a plastic hodoscope eSC surrounding the chambers.

To obtain undistorted $\mu \rightarrow e$ decay time spectra, muon transfers to higher- $\mathrm{Z}$ elements and also to deuterium must be avoided to levels below $10^{-5}$ which leads 
Fig. 1 Picture of the TPC developed at PSI, used as active muon stop target
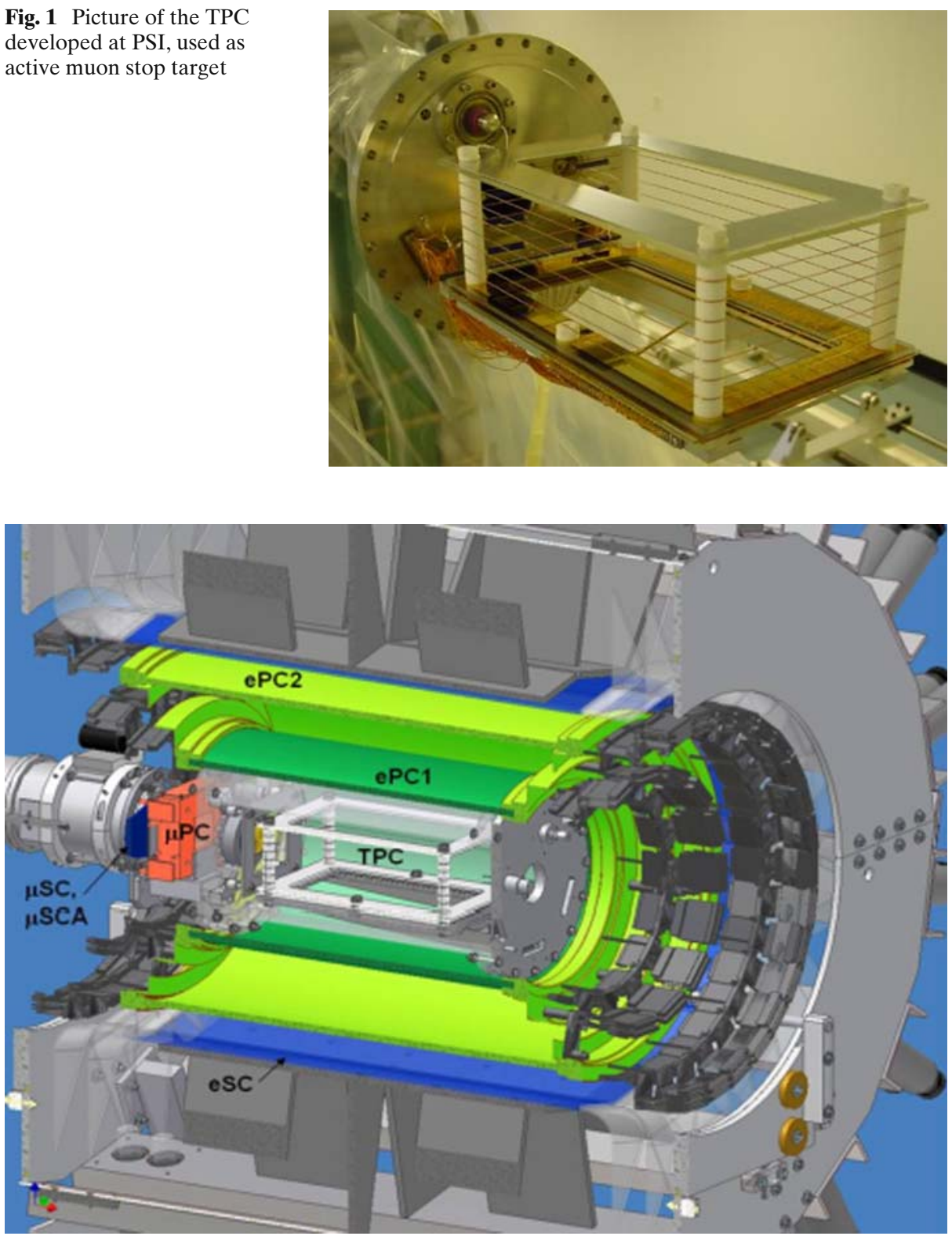

Fig. 2 Cut-out view of the MuCap experimental setup

to the stringent requirement of keeping impurities in the protium gas below $10^{-7}$. We have accomplished this requirement by constructing a special gas purification system [7]. Using active-carbon based cryo-adsorption pumps this system circulates the gas with a constant flow rate and pushes it through zeolite filters at liquid nitrogen temperature. The main impurity in the protium was identified to be water vapour and was kept below 20 ppb during the main runs. 
Fig. $3 \mu p$ lifetime spectra of the first MuCap run using different different cuts of the $\mu-e$ impact vertex

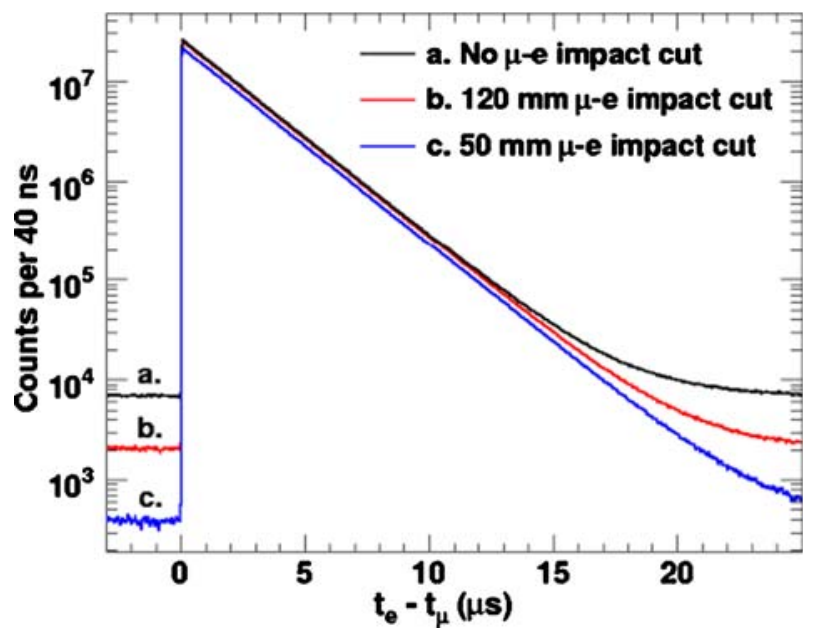

Since even a small presence of deuterium in the protium leads to significant muon transfers $\mu p+d \rightarrow \mu d+p^{\prime}$ followed by strong diffusion of the $\mu d$ atoms out of the TPC volume, we have constructed a novel isotope separation facility to remove all deuterium traces. The facility consists of a cryogenic column of $1.5 \mathrm{~m}$ height which separates the isotopes on the basis of their different vapour pressures. The hydrogen gas is liquefied by a cold head at the top of the column, and the liquid droplets run down and evaporate. This procedure strongly depletes the heavier isotope in the gas phase. In AMS measurements at ETH Zurich the deuterium depletion of our protium was determined to be below 6 ppb.

The MuCap experiment was run successfully during four independent data taking periods. A total statistics of nearly $2 \cdot 10^{10}$ good $\mu$-e events was accumulated which will allow us to reach the anticipated precision on $\Lambda_{S}$ of $<1 \%$ and on $\mathrm{g}_{P}$ of $\sim 5 \%$. The evaluation of our first run with about $10 \%$ of the total statistics was completed in 2007. Figure 3 shows the $\mu p$ lifetime spectra from this run using various selection criteria for the $\mu e$ impact vertex. We have published in [8] a first precise result of the $\mu p$ singlet capture rate as

$$
\Lambda_{S}^{\text {MuCap }}=\left(725.0 \pm 13.7_{\text {stat }} \pm 10.7_{\text {sys }}\right) s^{-1} .
$$

The main systematic error comes from uncertainties of impurity corrections and the physics of $p \mu p$ molecules. This result yields an induced pseudoscalar coupling constant

$$
g_{P}^{M u C a p}=7.3 \pm 1.1
$$

which is-after radiative corrections [9] -in good agreement with $\mathrm{g}_{P}^{\text {theory }}=8.26 \pm$ 0.23 [3]. From analysis with the full statistics we expect a two to three times better accuracy. The quality of the final result will also depend on the success of the special runs which we have made using specific small admixtures of $\mathrm{N}_{2}, \mathrm{H}_{2} \mathrm{O}$ and Ar gases to determine the corrections for impurities and for the formation of $p \mu p$ molecules. 
Fig. 4 Time development of the populations of $\mu d$ hyperfine states and $\mu \mathrm{He}^{3}$ atoms

Fig. 5 Cut-out view of the new setup with the Cryo-TPC in the center
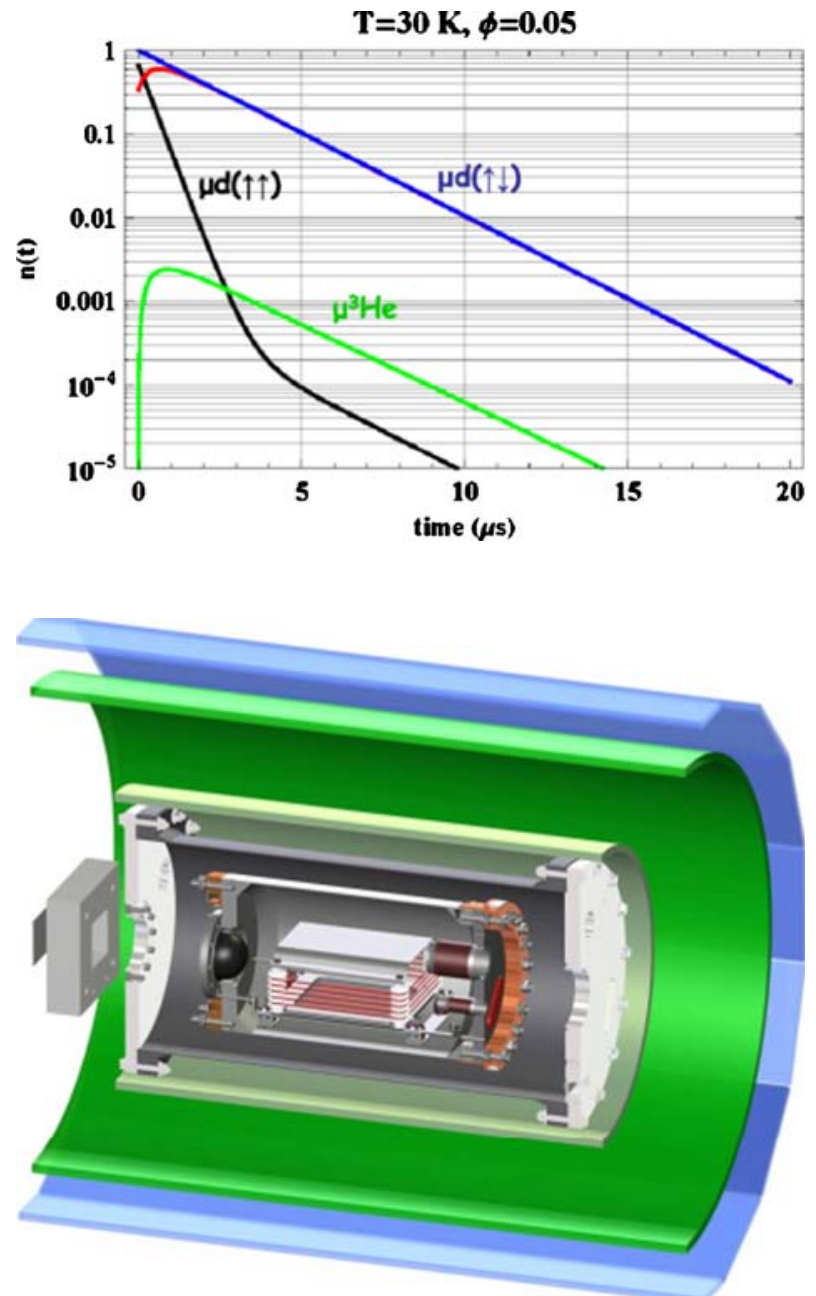

\section{The MuSun experiment}

The $\mu d$ capture experiment will adopt the same method of lifetime measurements as MuCap, and to a large part it will use the same equipment and electronics. In one respect, however, the apparatus needs a total redesign: the $\mu d$ measurements must be performed at low temperature, $\mathrm{T} \sim 30 \mathrm{~K}$, and at higher gas density compared to the MuCap experiment, $\phi \sim 0.05-0.10$. These conditions are dictated by the relatively slow spin-flip rate $\mu d^{F=3 / 2}+d \rightarrow \mu d^{F=1 / 2}+d^{\prime}$ with an effective rate $\Lambda_{3 / 2,1 / 2} \sim$ $3.7 \mu s^{-1}$ at $\phi=0.1[10,11]$, and because the $\mu d$ spin states can only be monitored at low temperature. The monitoring is done by observing the time distribution of $d \mu d$ fusion events. Figure 4 shows the time development of the $\mu d$ hyperfine states at $30 \mathrm{~K}, \phi=0.1$. The depopulation of $\mu d^{F=3 / 2}$ states is easily observable due to the phenomenon of resonant formation of $d \mu d$ molecules which is at low temperatures confined to the upper hyperfine level $\mathrm{F}=3 / 2[10,11]$. The $\mu H e^{3}$ population is also 
shown in Fig. 4, originating from $d \mu d$ fusion events followed by muon sticking to the $\mathrm{He}^{3}$ fusion product.

The new Cryo-TPC, designed to run at the required new target conditions, is presently in construction at Gatchina. Figure 5 gives a cut out view of the experimental setup. The TPC is embedded in an inner cylinder volume which can be cooled by a liquid Neon circuit. The outer cylinder acts now as insulation vacuum chamber for the cryogenic part and fits into the existing electron detector. The TPC collects the charges on a pad structure of size $10 \times 10 \mathrm{~cm}^{2}$. Over the vertical drift length of $8 \mathrm{~cm}$ an electrical field of $\sim 10 \mathrm{kV} / \mathrm{cm}$ shall be applied. Similar conditions were used in previous $\mu \mathrm{CF}$ experiments at PSI [11], although the chamber geometry was considerably smaller.

The purity of the deuterium gas is again of crucial importance. Due to the higher density and to expected larger transfer rates $\mu d \rightarrow \mu Z$ as compared to the MuCap experiment, impurity levels of $1 \mathrm{ppb}\left(\mathrm{N}_{2}, \mathrm{H}_{2} \mathrm{O}\right.$, etc) must be achieved and maintained. We expect, that the existing circulation system [7] will suffice in the low temperature environment. Several methods to monitor the impurities are under investigation. It will also be important to avoid any complications from HD kinetics. We have therefore utilized the MuCap isotope separation facility in the reversed mode and already removed the hydrogen- 1 isotope from the commercially bought deuterium gas.

In an initial run late 2008 we will test prototypes of the new TPC geometry to verify the expected performance. We will use several neutron counters to observe also the $d \mu d$ fusion neutrons, and we plan to determine the transfer rates to impurities. The commissioning the Cryo-TPC is scheduled in fall 2009. We plan to collect $\sim 3 \cdot 10^{10}$ good $\mu-e$ events of which one third will be with $\mu^{+}$beam to investigate systematic issues.

\section{References}

1. MuCap collaboration http://www.npl.uiuc.edu/exp/mucapture

2. MuSun collaboration http://www.npl.uiuc.edu/exp/musun

3. Bernard, V., et al.: J. Phys. G28, R1 (2002)

4. Ando, S., et al.: Phys. Lett. B533, 25 (2002)

5. Chen, J.W., et al.: Phys. Rev. C72, 061001 (2005)

6. Bardin, G., et al.: Nucl. Phys. A352, 365 (1981)

7. Ganzha, V.A., et al.: Nucl. Instr. Meth. A578, 485 (2007)

8. Andreev, V.A., et al.: Phys. Rev. Lett. 99, 032002 (2007)

9. Czarnecki, A., et al.: Phys. Rev. Lett. 99, 032003 (2007)

10. Zmeskal, J., et al.: Phys. Rev. A42, 1165 (1990)

11. Petitjean, C., et al.: Hyperfine Interactions 118, 127 (1999) 